\title{
Two-channel model based adaptive schlieren detection algorithm for BOS system
}

\author{
LIU Han ${ }^{1}$, ZHANG Yanmei ${ }^{1, *}$, ZHAO Baojun ${ }^{1}$, GUO Haichao ${ }^{2}$, and ZHAO Boya ${ }^{1}$ \\ 1. School of Information and Electronics, Beijing Institute of Technology, Beijing 100081, China; \\ 2. National Key Laboratory of Science and Technology on Space Microwave, China Academy of Space Technology, \\ Xi'an 710100, China
}

\begin{abstract}
A schlieren detection algorithm is proposed for the ground-to-air background oriented schlieren (BOS) system to achieve high-speed airplane shock waves visualization. The proposed method consists of three steps. Firstly, image registration is incorporated for reducing errors caused by the camera motion. Then, the background subtraction dual-model single Gaussian model (BS-DSGM) is proposed to build a precise background model. The BS-DSGM could prevent the background model from being contaminated by the shock waves. Finally, the twodimensional orthogonal discrete wavelet transformation is used to extract schlieren information and averaging schlieren data. Experimental results show our proposed algorithm is able to detect the aircraft in-flight and to extract the schlieren information. The precision of schlieren detection algorithm is 0.96 . Three image quality evaluation indices are chosen for quantitative analysis of the shock waves visualization. The white Gaussian noise is added in the frames to validate the robustness of the proposed algorithm. Moreover, we adopt two times and four times down sampling to simulate different imaging distances for revealing how the imaging distance affects the schlieren information in the BOS system.
\end{abstract}

Keywords: background model, background oriented schlieren (BOS), schlieren detection, wavelet decomposition.

DOI: $10.21629 / J S E E .2019 .02 .04$

\section{Introduction}

Visualization techniques such as schlieren, photography, shadowgraphy and interferometry have been used in flow field recognition and analysis for many decades. A simplified schlieren technology called the background oriented schlieren (BOS) system was proposed by Meier in 1999 [1]. Unlike conventional visualization techniques, the BOS system adopts the image-processing algorithm in the system to simplify optical system settings and achieve outdoor large-scale flow imaging and in-flight measurement. The BOS imaging system has been applied to many areas, such

Manuscript received March 16, 2018

*Corresponding author. as quantitative flow field analysis of aerodynamic [2,3], predicting landing position of aircraft, analysis of aircraft accident [4] and so on. In this study, a method for the BOS imaging system is proposed for the BOS imaging system to achieve a ground to air real-time surveillance system. There are two fundamental functions in this system: warning the flying objects detected and visualizing the shock waves for obtaining detailed information of the aircraft.

The BOS imaging system consists of an adequate resolution camera, a background with speckles and a flow field. This technique relies on visualization of background speckle pattern shifts between the image pairs, which presents the refractive index caused by high-speed motion objects. The cross-correlation algorithm and the optical flow algorithm are applied in the BOS system for calculating image displacements. The cross-correlation algorithm relies on the offset of the window. Consequently, the averaged offsets of the interrogation window induce serious distortion [5]. Recently, a set of computer vision based optical flow algorithms are applied in the BOS system to visualize shock waves, and get a better performance compared with cross-correlation algorithms [6,7]. However, the pixel-level based optical flow algorithm is a timeconsuming process and sensitive to noises and luminance variations. Megapixel-range BOS images and complex optical flow algorithms make the system difficult to reach the real-time requirement.

In this paper, a schlieren detection and schlieren visualization algorithm for the BOS system is proposed. Different from the cross-correlation algorithm and the optical flow algorithm, we visualize the schlieren by object detection and tracking. The image registration processing is incorporated for reducing errors caused by camera motion, such as translation, rotation and tilting. Besides, a algorithm named background subtraction dual-model single Gaussian model (BS-DSGM) is proposed for high-speed 
motion. This algorithm adopts updating the background and foreground models online, which makes the background model insensitive to the foreground model. Considering the discontinuities and high-frequency variations of the BOS data [8], the two-dimensional orthogonal discrete wavelet transformation is adopted to extract the highfrequency schlieren information of the shock waves.

\section{The proposed method}

The proposed schlieren detection algorithm for the BOS system consists of three steps: the BOS image registra- tion, the schlieren detection and the schlieren information extraction. The image registration reduces the error caused by camera motion and improves the accuracy of detection results. Then, the statistical model with the adaptive factor makes the model adjust background changes and the DSGM makes the background model insensitive to the shock waves and illumination variations. The twodimensional orthogonal discrete wavelet transformation is applied to extract high frequency schlieren information and averaging of schlieren data. Fig. 1 shows the framework of the proposed method.

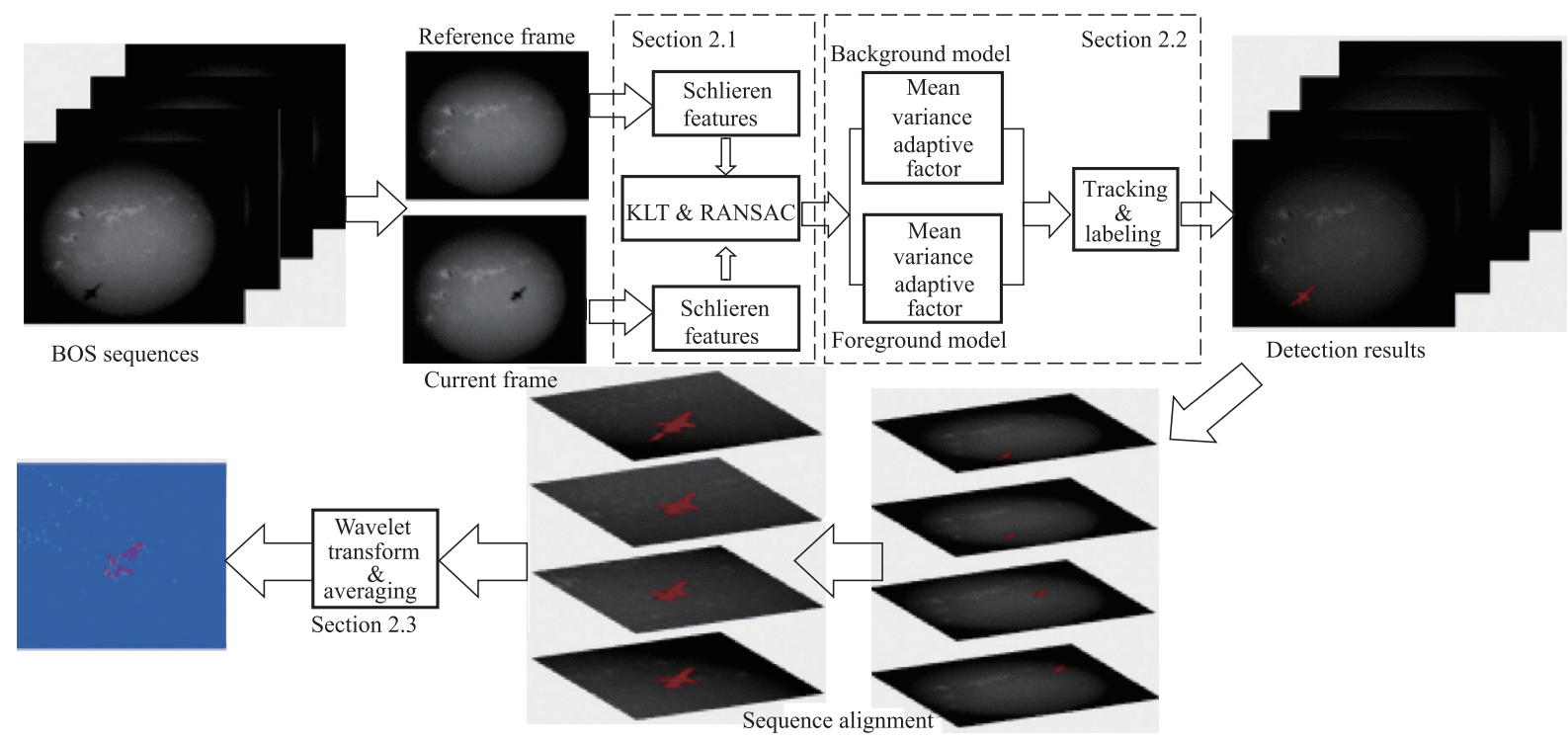

Fig. 1 Framework of motion detection and tracking algorithm for BOS system

\subsection{BOS images registration}

Elsinga et al. [9] reminded that problems related to unsteady displacement of the tunnel and vibration are intrinsic to the supersonic wind tunnel environment. The background motion problem generated by the flow is intrinsic in the BOS system and makes the background unstable [10]. To overcome this problem, image registration processing is very necessary to eliminate the effects of the background motion problem.

For BOS sequences, the initial step is selecting a pass with the target aircraft in the camera views. The first frame of the selected pass is a pure background sequence regarded as reference frame. The stack of subsequent frames is registered to the reference frame by the KLT algorithm, which calculates spatial intensity gradients of adjacent frames at four schlieren features near the corners of the grid [11]. The KLT algorithm estimates the local motion of the scene by calculating the movements between time $t$ and time $t+1$. The movements between the reference frame and current frame are kept with homograph matrix. Then, the RANSAC algorithm is applied to acquire homograph matrix $\boldsymbol{H}_{t: t+1}$, which warps all pixels in time $t+1$ to pixels in time $t$ through a perspective transform [12].

There are several advantages to apply image registration algorithm for the BOS system to achieve schlieren detection. Firstly, image registration eliminates the errors of camera movement, the movement between the aircraft and solar facula, and insures that the displacement between aircraft and background is not offset. Secondly, image registration reduces false labelling of distorted pixels and improves the accuracy of schlieren detection. Moreover, BOS image registration makes the background model to be stationary.

\subsection{Schlieren detection}

The idea of our schlieren detection algorithm comes from: if the specific aircraft is detected, then schlieren could be detected. The BS-DSGM algorithm is proposed for building background and foreground models respectively. 
Starting with detection of moving objects, a guided filter is adopted for BOS images pre-processing, which is a local linear filter to achieve filtering by guided images to reduce the noises and maintain the edge information. The output of guided filter images have more structure information and less smoothness [13].

\subsubsection{Background model}

The BOS images have large image size, a uncomplicated background with one flying object. However, a typical background subtraction algorithm fails in modelling a perfect background model due to the shock waves generated. The shock waves in the image represent image distortions and make the background unstable. Therefore, an improved BS-DSGM with an adaptive factor is adopted in this paper. In order to reduce the computation load, images are divided into four grids with an $N \times N$ size. Assume that each pixel in the grid obeys the Gaussian distribution [14], which has one mean and one variance. The major motion fits the Gaussian distribution considered as the background, and the outliers of the pixel value greater than the threshold are defined as moving objects. Moreover, the grids which have many outlier pixels or the values of the mean and variance vary greatly which implies the motion objects inside the grid. In this algorithm, we define an adaptive factor $r$ varying over temporal domain for the unstable background to estimate a sufficient statistics model and it improves adaptation of the background model and reduces detected objects. The current background model is updated from the previous frame. The online mechanism of the single Gaussian model is as follows:

$$
\begin{gathered}
\mu_{B, m}^{(t)}=r_{B, m}^{(t)} \mu_{B, m}^{(t-1)}+\left(1-r_{B, m}^{(t)}\right) G_{m}^{(t)} \\
\sigma_{B, m}^{2(t)}=r_{B, m}^{(t)} \sigma_{B, m}^{2(t-1)}+\left(1-r_{B, m}^{(t)}\right) V_{m}^{(t)} \\
r_{B, m}^{(t)}=\frac{r_{B, m}^{(t-1)}}{(c+1) r_{B, m}^{(t-1)}}
\end{gathered}
$$

where $\mu_{B, m}^{(t)}$ and $\sigma_{B, m}^{2(t)}$ are the mean and the variance of the background scene in the grid $m$ at time $t . r_{B, m}^{(t)}$ is an adaptive factor and smaller value means faster adaptive rate. The adaptive factor is defined by a constant $c$, which is used for estimating the statistics model. $G_{m}^{(t)}$ and $V_{m}^{(t)}$ are defined as

$$
\begin{gathered}
G_{m}^{(t)}=\frac{1}{\left|g_{m}\right|} \sum_{n \in g_{m}} I_{n}^{(t)} \\
V_{m}^{(t)}=\max \left(\mu_{m}^{(t)}-G_{m}^{(t)}\right)^{2} .
\end{gathered}
$$

For each grid, we assign the pixel value in the grid $m$ as $g_{m}$, and the pixel intensity of the pixel $n$ at time $t$ as $I_{n}^{(t)}$.

\subsubsection{Foreground model}

From the results of the algorithms comparison, we find that one SGM algorithm (Kwang-SGM) works well for object detection, but the background model is contaminated by the shock waves. The reason for this phenomenon is that the background model describes some portions of the foreground in one SGM as shown in Fig. 2. To solve this problem, we construct two SGMs for each grid: one SGM is used for building the background model and another one for building the foreground model. Fig. 2 illustrates the effects of one SGM and two SGMs for each grid.

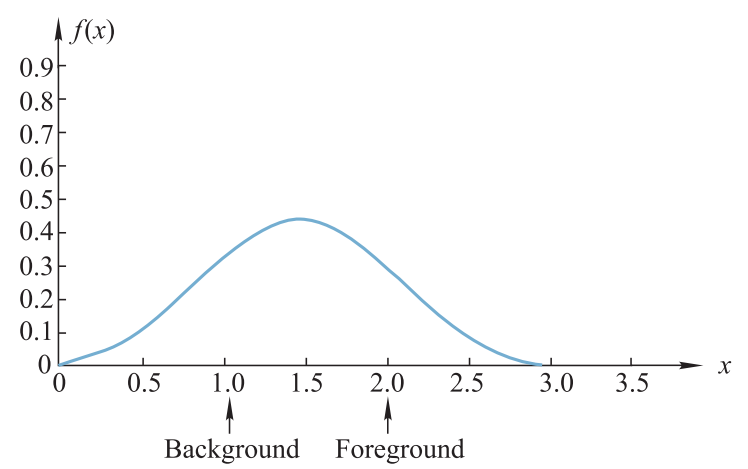

(a) One SGM

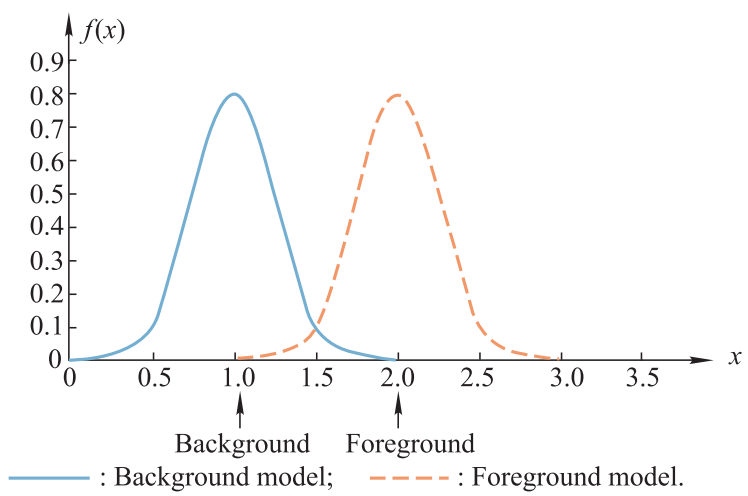

(b) Two SGMs

Fig. 2 Illustration of one SGM and two SGMs

We define the pixels which are outlier of the Gaussian distribution and greater in value than the threshold of the mean as foreground pixels. The foreground model to learn the background subtraction results is

$$
\left(G_{m}^{(t)}-\mu_{m}^{(t)}\right)^{2} \geqslant 3 \sigma_{m}^{(t)}
$$

The SGM for the grid $m$ of the foreground model is updated as follows:

$$
\begin{gathered}
\mu_{F, m}^{(t)}=r_{F, m}^{(t)} \mu_{F, m}^{(t-1)}+\left(1-r_{F, m}^{(t)}\right) G_{m}^{(t)} \\
\sigma_{F, m}^{2(t)}=r_{F, m}^{(t)} \sigma_{F, m}^{2(t-1)}+\left(1-r_{F, m}^{(t)}\right) V_{m}^{(t)} \\
r_{F, m}^{(t)}=\frac{r_{F, m}^{(t-1)}}{(c+1) r_{F, m}^{(t-1)}}
\end{gathered}
$$


The adaptive factor is defined for the foreground model due to the temporary nature. The meanings of the parameters in the foreground model are the same as those in the background model. We define the variance $\sigma_{F}$ greater than $\sigma_{B}$.

\subsubsection{Tracking and labeling}

The framework of the proposed schlieren detection algorithm combines the detection algorithm and the tracking algorithm [15]. After the motion objects are detected, we extract the features of objects for further aircraft trajectory tracking and prediction. The tracking algorithm is a maximization problem. Likelihood of the model is defined by using the likelihood of the foreground model.

$$
L_{F, n}=\mathcal{L}_{N}\left(\mu_{F, m}, \sigma_{F, m}^{2}, I_{n}\right)
$$

where $L_{F, n}$ represents log-likelihood for the $n$th pixel of the foreground model. Hypothesized position of the target object is defined as $H_{T}$.

$$
\widehat{H}_{T}=\operatorname{argmax} \sum_{n} L_{F, n}\left(H_{T}\right) .
$$

The tracking problem finds the estimated target position $\widehat{H}_{T}$ as defined in (11). Pixel-wise likelihood for the foreground model is designed to provide a better explanation and background pixels do not interfere with the estimation of the motion objects in the foreground.

\subsection{Schlieren information extraction}

Another main idea of our proposed method is performing wavelet decomposition to extract high-frequency schlieren information and averaging of schlieren data. Orthogonal wavelet transform represents the time-frequency of signal in wavelet domain, and it provides good time resolution for high frequency information and good frequency resolution for low frequency information [16-18]. Images are decomposed into several wavelet components based on various frequency bandwidths. In addition, decomposed frequency bandwidths can be constructed by orthogonal inverse wavelet transform and quantitative information can be obtained [19]. The two-dimensional orthogonal discrete wavelet transform is adopted in this paper, which decomposes the schlieren data into different frequency components. The wavelet decomposition in this paper is used to extract high frequency schlieren information generated by high-speed aircraft motions. The two-dimension orthogonal discrete wavelet transform is an orthogonal transform and deduced by multiplication of matrices:

$$
\boldsymbol{V}=\sum_{i=1}^{n} \boldsymbol{\psi}^{\mathrm{T}} \boldsymbol{X} \boldsymbol{\psi}
$$

where $\boldsymbol{X}$ is a two-dimensional data matrix. $\boldsymbol{V}$ and $\psi$ denotes discrete wavelet coefficients, $\boldsymbol{\psi}^{\mathrm{T}}$ is a transposed matrix.

In this paper, Daubechies wavelet basis has been chosen. The steps of constructing compactly supported orthogonal wavelet are as follows:

Step 1 Setting $N \geqslant 2$.

Step 2 Using trigonometric identity equation to solve coefficients $q_{n}$ and $Q_{n}(Z)$.

$$
\left|Q_{N}\left(\mathrm{e}^{\mathrm{i} \omega}\right)\right|^{2}=P_{N}\left(\sin ^{2} \frac{\omega}{2}\right)
$$

where $Q_{N}\left(\mathrm{e}^{\mathrm{i} \omega}\right)$ and $P_{N}(y)$ are algebraic polynomials:

$$
\begin{aligned}
& P_{N}(y)=\sum_{k=0}^{N-1} C_{N+k}^{k} y^{k} \\
& Q_{N}\left(\mathrm{e}^{\mathrm{i} \omega}\right)=\sum_{n=0}^{N-1} q_{n} \mathrm{e}^{\mathrm{i} \omega} .
\end{aligned}
$$

Step 3 According to the polynomial of $m_{0}(z)$, we obtain series $\left\{h_{n}\right\}$ :

$$
m_{0}(z)=\left(\frac{1+z}{2}\right)^{N} Q_{N}(z) .
$$

Series $\left\{h_{n}\right\}$ satisfies the following conditions:

$$
\begin{gathered}
\sum_{n \in \mathbf{Z}} h_{n} \bar{h}_{n-2 k}=2 \delta_{0 k} \\
\sum_{n \in \mathbf{Z}} h_{n}=2 \\
\sum_{n \in \mathbf{Z}} h_{2 n}=\sum_{n \in \mathbf{Z}} h_{2 n+1}=1 .
\end{gathered}
$$

Step 4 According to the scale function, we calculate the iterative solution $\varphi(x)$ :

$$
\varphi(x)=\sum_{n \in \mathbf{Z}} h_{n} \varphi(2 t-n) .
$$

Step 5 The orthogonal wavelet $\psi(x)$ can be obtained by

$$
\psi(x)=\sum_{k \in \mathbf{Z}} \bar{h}_{1-n} \varphi(2 t-n) .
$$

The data are decomposed into four kinds of frequency components, referred to as LL, LH, HL and HH. The four transfer functions $V\left(\omega_{x}, \omega_{y}\right)$ of the 2D wavelet at the octave $i$ are shown in below:

$$
\begin{aligned}
V_{\mathrm{LL}}^{i}\left(\omega_{x}, \omega_{y}\right) & =H_{\varphi}^{i}\left(\omega_{x}\right) H_{\varphi}^{i}\left(\omega_{y}\right) \\
V_{\mathrm{LH}}^{i}\left(\omega_{x}, \omega_{y}\right) & =H_{\varphi}^{i}\left(\omega_{x}\right) H_{\psi}^{i}\left(\omega_{y}\right) \\
V_{\mathrm{HL}}^{i}\left(\omega_{x}, \omega_{y}\right) & =H_{\psi}^{i}\left(\omega_{x}\right) H_{\varphi}^{i}\left(\omega_{y}\right) \\
V_{\mathrm{HH}}^{i}\left(\omega_{x}, \omega_{y}\right) & =H_{\psi}^{i}\left(\omega_{x}\right) H_{\psi}^{i}\left(\omega_{y}\right)
\end{aligned}
$$


where $H_{\varphi}^{i}(\omega)=\frac{\varphi\left(2^{i}\right)}{\varphi(\omega)}$ and $H_{\psi}^{i}(\omega)=\frac{\psi\left(2^{i}\right)}{\varphi(\omega)}$.

\section{Experimental results}

\subsection{Simulation}

Our validation datasets are collected by the backgroundoriented schlieren using the celestial objects system (CaKEBOS) and the data could be downloaded from the NASA website [20]. For the BOS system, long-focal lens with higher resolution of camera is chosen. Thus, more information can be captured and the shock waves are visualized more clearly. The ideal background image has highcontrast, random and suitable sizes speckles. The sensitivity of the BOS system is related to the distance $Z_{B}$; longer distance between the flow filed and the background implies greater sensitivity. $\Delta y$ is the pixel shift; smaller image displacement means higher accuracy of the BOS system. The experimental setup of the CaKEBOS system is shown in Fig. 3.

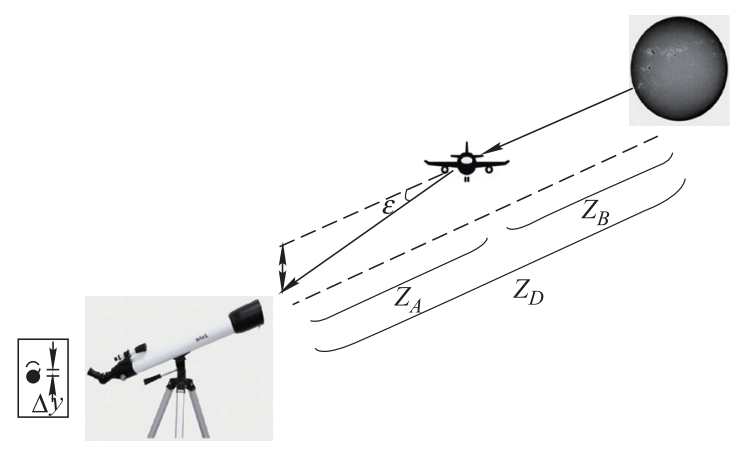

Fig. 3 CaKEBOS system's experimental setup

The CaKEBOS imaging system consists of a groundbased imaging system, the sun regarded as background and an aircraft. The ground-based BOS imaging system is designed by NASA, which is composed of a vixen telescope, a Lunt B1200 Calcium-K filter, a $2 \times$ focal extender and a Hispec high-speed digital camera for data recording [20]. $Z_{A}$ equals $50 \mathrm{~km}$, and $Z_{D}$ is approximately equal to 150 million $\mathrm{km}$. The frame size of the original data is $1280 \times 1024$. The size of the compressed video we obtain from the website is $1200 \times 700$.

The proposed algorithm is implemented by using Microsoft Visual, C++ and Matlab 2013b on the Windows 7 working environment, and the computer configuration is Intel $\AA$ Corel $^{\mathrm{TM}}$ i5-4590U CPU with $3.30 \mathrm{GHz}$ and 4.00 GB system RAM. For parameter setting, we set initial adaptive factor $r$ equal to 0.02 . Smaller $\sigma$ implies more accuracy of the background model. For the wavelet decomposing, we choose the Daubechies wavelet basis and $N$ equals 4.

\subsection{Results and analysis}

\subsubsection{Detection results of BOS data}

We compare our proposed BS-DSGM against two other methods, the Kwang-SGM algorithm [14] and the visual background extractor (VIBE) algorithm [21]. The results of aircraft object detection are binary images: the foreground is white and the background is black. As results shown in Fig. 4, the results of the BS-DSGM are better than those of the Kwang-SGM and the VIBE algorithm. The background model of the Kwang-SGM algorithm is contaminated by shock waves, and the VIBE algorithm detects incomplete aircrafts. As we discuss in Section 2.2.2, the reason is that the portions of foreground pixels are classified as the background. The BS-DSGM algorithm builds the precise background model and foreground model respectively; hence it prevents the background from being contaminated by the shock waves.
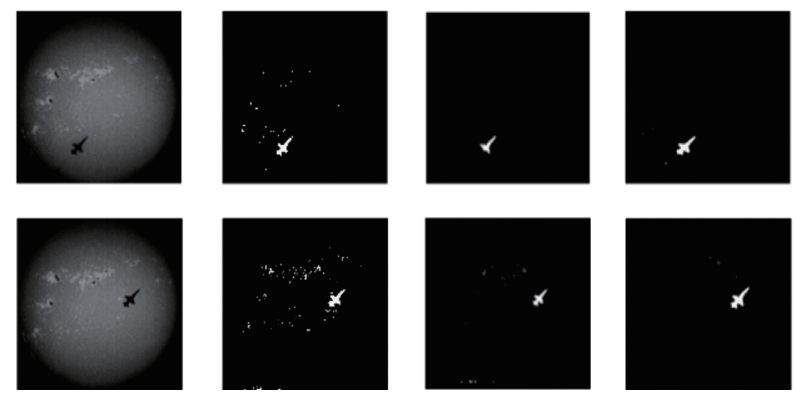

VIBE

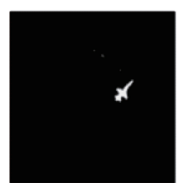

BS-DSGM

Fig. 4 Comparison of detection results between BS-DSGM algorithm and BS-SGMs algorithm

\subsubsection{Quantitative analysis of detection results}

The performance of the proposed detection algorithm is quantitative analysis by precision and the recall curve is shown in Fig. 5.

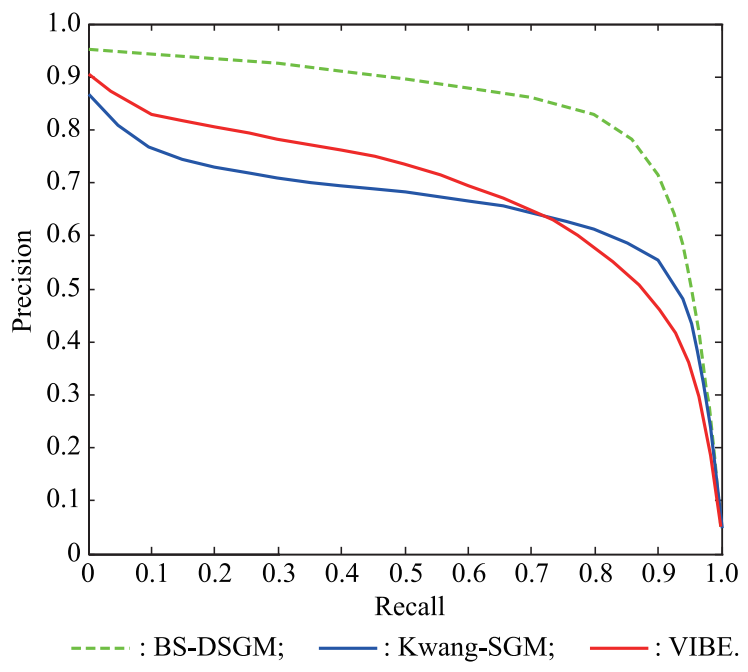

Fig. 5 Detection performance of the proposed algorithm 
The precision and recall are calculated by

$$
\begin{gathered}
\text { Precision }=\frac{T P}{T P+F P} \\
\text { Recall }=\frac{T P}{T P+F N}
\end{gathered}
$$

where $T P, F P$ and $F N$ are defined in Table 1. The proposed algorithm with the uncomplicated background model reduces the computation loads. Moreover, the background model is updated with the current frame. From Fig. 5 , we observe the precision of detection is 0.96 . Our precise background and foreground models reduce the number of falsely detected objects.

Table 1 Precision and recall definition

\begin{tabular}{ccc}
\hline & Actual object & Non-object \\
\hline Detected & $T P$ & $F P$ \\
Not detected & $F N$ & $T N$ \\
\hline
\end{tabular}

\subsubsection{Quantitative analysis of shock waves visualization}

The result of the proposed algorithm is shown in Fig. 1. 80 frames are chosen to generate the time-averaged images. The result limited by resolution degrades due to the compressed video. To further evaluate the quality of our proposed algorithm, we choose three kinds of image quality evaluation indices, the structural similarity index measurement (SSIM), the peak signal to noise ratio (PSNR) and the mean squared error (MSE), to make a comparison between the cross-correlation algorithm and the optical flow algorithm. The comparison results are shown in Table 2.

Table 2 Comparison of performance for schlieren extraction

\begin{tabular}{cccc}
\hline Method & BS-DSGM & $\begin{array}{c}\text { Cross } \\
\text { correlation [3] }\end{array}$ & $\begin{array}{c}\text { Optical } \\
\text { flow [4] }\end{array}$ \\
\hline MSE & 100 & 400 & 320 \\
PSNR/dB & 29 & 15 & 17 \\
SSIM & 0.72 & 0.33 & 0.42 \\
\hline
\end{tabular}

The MSE is variance with the reference image and current image; the value closer to zero means better. The PSNR is the most widely used objective evaluation index. The range of video compression is between $30 \mathrm{~dB}$ and $50 \mathrm{~dB}$ and the higher is better [22]. The SSIM is used for measuring the similarity between two images. The value range of the SSIM is $[0,1]$, and the higher implies better as well [23]. Table 2 demonstrates the performance of the BSDSGM algorithm precedes the other two algorithms. The accuracy of the cross-correlation algorithm is based on the size of the interrogation window. The iterative processes produce large computation and average over interrogation window size makes image distorted. The simulation time of the cross-correlation algorithm is $26.55 \mathrm{~s}$. Moreover, the optical flow algorithm is complicated, susceptible to noise interference and cannot achieve real-time tracking unless there is strong hardware support. The simulation time of the optical flow algorithm is $47 \mathrm{~s}$. Our proposed algorithm has the shortest calculation time of $11.54 \mathrm{~s}$.

\subsubsection{Anti-interference analysis}

To validate the robustness of our proposed algorithm, we add Gaussian white noise into the original video frames. The Gaussian white noise added will impact accuracy of image registration, motion object detection and effectiveness of schlieren extraction. The four curves shown in Fig. 6 with different colors represent four videos. The SSIM is decreased by increasing the intensity of Gaussian white noise. However, from Fig. 6, with the noise intensity between $20 \mathrm{~dB}$ and $30 \mathrm{~dB}$, the SSIM of our proposed algorithm is higher than that of the cross-correlation algorithm and the optical flow algorithm shown in Table 1. Hence, our proposed algorithm is able to realize anti-interference and has good robustness.

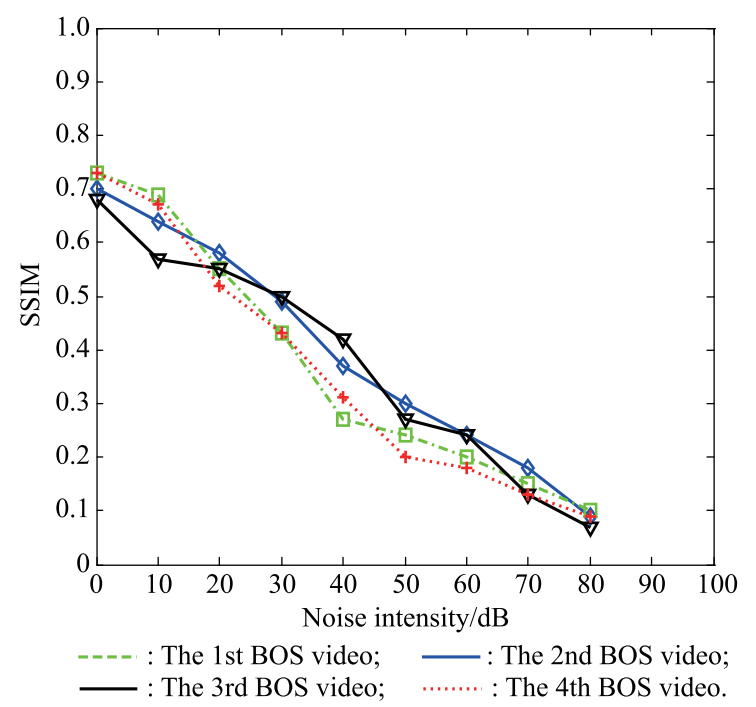

Fig. 6 Performance of the proposed algorithm with randomly adding white noise

\subsubsection{Effects of imaging distance}

To evaluate the influence of the imaging distance on the proposed algorithm, we adopt two times down sampling and four times down sampling to simulate imaging distances of the BOS system. The SSIM parameter is chosen to evaluate the performance. The validation datasets are the same as those in Section 3.2.3. For four times down sampling, the results of the shock waves visualization are not very good due to the long distance. From Table 3, our proposed algorithm has good performance in BOS systems with different imaging distances. The results are in the greatest sensitivity BOS system. 
Table 3 Performance of the proposed method with different imagery distances

\begin{tabular}{ccccc}
\hline Video & \multicolumn{4}{c}{ SSIM } \\
\hline Original video & 0.72 & 0.68 & 0.69 & 0.71 \\
Two times down sampling video & 0.60 & 0.58 & 0.59 & 0.57 \\
Four times down sampling video & 0.49 & 0.47 & 0.50 & 0.44 \\
\hline
\end{tabular}

\section{Conclusions}

In this paper, we propose a schlieren detection algorithm for the BOS imaging system to achieve the ground to air real-time surveillance system. The ground-to-air BOS imaging system is applied to capture the schlieren of inflight aircrafts, then image-processing algorithms are used to achieve schlieren detection and visualization. In order to reduce the errors caused by camera motion, the KLT and RANSAC algorithms are applied for the image registration processing. Then, a algorithm named BS-DSGM is proposed for modeling background scene and foreground. The highlight of this algorithm is that it prevents the background model from being contaminated by the shock waves. After calculating the detection and tracking results of the aircraft, we perform the two-dimensional orthogonal discrete wavelet transformation to extract schlieren information of the shock waves. The proposed method helps the BOS system achieve shock waves surveillance in real time. From the results of validation datasets, the proposed algorithm is able to detect in-flight aircrafts and makes the BOS system more accurate and sensitive to aircrafts.

\section{References}

[1] MEIER G E A. New optical tools for fluid mechanics. Sadhana, 1998, 23(5/6): $557-567$.

[2] RAFFEL M, HEINECK J T, SCHAIRER E, et al. Background-oriented schlieren imaging for full-scale and inflight testing. Journal of American Helicopter Society, 2014, 59(1): $1-9$.

[3] WEINSTEIN L M, KATHRYN S, VIEIRA G J. Visualization and image processing of aircraft shock wave structures. Proc. of the 1st Pacific Symposium on Flow Visualization and Image Processing, 1997: 23-26.

[4] SUEISHI T, ISHII M, ISHIKAWA M. Tracking backgroundoriented schlieren for observing shock oscillation of transonic flying objects. Applied Optics, 2017, 56(13): 3789.

[5] LIU T, MERAT A, MAKHMALBAF M H M, et al. Comparison between optical flow and cross-correlation methods for extraction of velocity fields from particle images. Experiments in Fluids, 2015, 56(8): 166.

[6] HEINECK J T, BANKS D, SCHAIRER E T, et al. Background oriented schlieren (BOS) of a supersonic aircraft in flight. Proc. of the AIAA Flight Test Aviation Conference, 2016: $13-$ 17.

[7] SMITH N T, HEINECK J T, SCHAIRER E T. Optical flow for flight and wind tunnel background oriented schlieren imaging. Proc. of the AIAA Aerospace Sciences Meeting, 2017: 472.

[8] HARGATHER M J, SETTLES G S. Natural-background oriented schlieren imaging. Experiments in Fluids, 2010, 48(1): $59-68$.
[9] ELSINGA G E, OUDHEUSDEN B W, SCARANO F, et al. Assessment and application of quantitative schlieren methods calibrated color schlieren and background oriented schlieren. Experiments Fluids, 2004, 36(2): 309-325.

[10] SETTLES G S, HARGATHER M J. A review of recent developments in stiller and shadowgraph techniques. Measurement Science \& Technology, 2017, 28(4): 42001.

[11] LUCAS B D, KANADE T. An iterative image registration technique with an application to stereo vision. Proc. of the International Joint Conference on Artificial Intelligence, 1981: $674-679$.

[12] FISCHLERM A, BOLLESR C. Random sample consensus: a paradigm for model fitting which application to image analysis and automated cartography. Communication of the AMC, 1981, 24: 381 - 395.

[13] HE K, SUN J, TANG X. Guided image filtering. IEEE Trans. on Software Engineering, 2013, 35(6): $1397-1409$.

[14] YIK M, YUN K, KIM S W, et al. Detection of moving object with non-stationary cameras in $5.8 \mathrm{~ms}$ : bringing motion detection to your mobile device. Proc. of the IEEE Conference Computation Vision and Pattern Recognition Workshops, 2013, 35(6): $27-34$.

[15] YI K M, JEONG H, LEE B, et al. Visual tracking in complex scenes through pixel-wise tri-modeling. Machine Vision and Applications, 2015, 26(2/3): 205-217.

[16] YUAN J, SCHNÖRR C, MÉMIN E. Discrete orthogonal decomposition and variational fluid flow estimation. Journal of Mathematical Imaging and Vision, 2007, 28(1): 67-80.

[17] RINOSHIKA A, ZHOU Y. Orthogonal wavelet multiresolution analysis of a turbulent cylinder wake. Journal of Fluid Mechanics, 2005, 524: 229-248.

[18] DÉRIAN P, HÉAS P, HERZET C, et al. Wavelet-based fluid motion estimation. Proc. of the International Conference on Scale Space and Variational Methods in Computer Vision, 2011: $737-748$.

[19] ZHENG Y, FUJIMOTO S, RINOSHIKA A. Two-dimensional wavelet multi-resolution analysis on turbulent structures of dune wake. Proc. of the International Conference on Wavelet Analysis and Pattern Recognition, 2014: 153-158.

[20] HILL M A, HAERING E A. Ground-to-air visualization using solar calcium-k line background-oriented schlieren. https://ntrs.nasa.gov/archive/nasa/casi.ntrs.nasa.gov/20160007969.pdf

[21] BARNICH O, DROOGENBROECK M V. ViBe: a powerful random technique to estimate the background in video sequences. Proc. of the IEEE International Conference on Acoustics, Speech and Signal Processing, 2009: 945 - 948.

[22] HUYNH-THU Q, GHANBARI M. Scope of validity of PSNR in image/video quality assessment. Electronics Letters, 2008, 44(13): $800-801$.

[23] WANG Z, BOVIK A C, SHEIKH H R, et al. Image quality assessment: from error visibility to structural similarity. IEEE Trans. on Image Processing, 2004, 13(4): 600-612.

\section{Biographies}

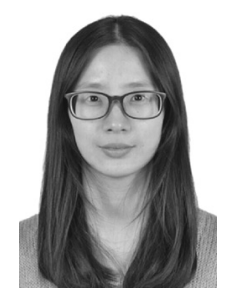

LIU Han was born in 1989. She received her B.S. degree from University of Sussex at the United Kingdom in 2013. Currently, she is pursuing the $\mathrm{Ph} . \mathrm{D}$. degree at the School of Electrical and Information Engineering, Beijing Institute of Technology. Her research interests include BOS system, object detection, and flow field visualization. E-mail: 3120140335@bit.edu.com 


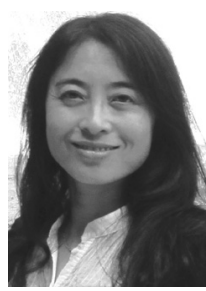

ZHANG Yanmei was born in 1967. She received her Ph.D. degree in electromechanical engineering from Beijing Institute of Technology in 2010. Since 1995, she has worked in Beijing Institute of Technology as a professor and a Ph.D. supervisor. Her interests include laser detection, BOS system, and compressed sensing.

E-mail:0726zym@bit.edu.cn

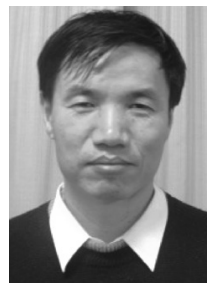

ZHAO Baojun was born in 1960. He received his Ph.D. degree in electromagnetic measurement technology and equipment from Harbin Institute of Technology in 1996. From 1996 to 1998, he was a postdoctoral fellow at Beijing Institute of Technology. His main research interests include image/video coding, image recognition, infrared/laser signal processing, and parallel signal processing.

E-mail: zbj@bit.edu.cn

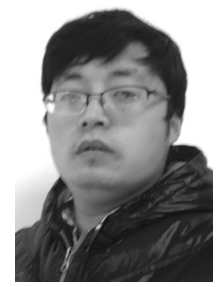

GUO Haichao was born in 1983. He received his B.S. degree in optical engineering from University of Electronic Science and Technology in 2006. From 2009 till now, he studies satellite free space coherent optical communication and laser imaging in $\mathrm{Na}$ tional key Laboratory of Science and Technology on Space Microwave and the School of Information and Electronics of Beijing Institute of Technology School.

E-mail: haizi0919@163.com

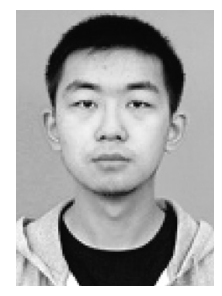

ZHAO Boya was born in 1990. He received his B.S. degree from the School of Electrical Engineering and Information, Hebei University of Technology, Tianjin, China, in 2013. He is currently pursuing his $\mathrm{Ph} . \mathrm{D}$. degree at the School of Information and Electronics, Beijing Institute of Technology, Beijing, China. His current research interests include object detection, object tracking and machine learning.

E-mail: zhaoboya@bit.edu.cn 